\title{
Some Remarks on Wittgenstein's Philosophy of Mathematics
}

\author{
Richard Startup \\ Honorary Research Fellow, Swansea University, Swansea, UK \\ Email: r.startup@swansea.ac.uk
}

How to cite this paper: Startup, R. (2020). Some Remarks on Wittgenstein's Philosophy of Mathematics. Open Journal of Philosophy, 10, 45-65.

https://doi.org/10.4236/ojpp.2020.101005

Received: November 6, 2019

Accepted: December 31, 2019

Published: January 3, 2020

Copyright $\odot 2020$ by author(s) and Scientific Research Publishing Inc. This work is licensed under the Creative Commons Attribution International License (CC BY 4.0). http://creativecommons.org/licenses/by/4.0/

\begin{abstract}
Drawing mainly from the Tractatus Logico-Philosophicus and his middle period writings, strategic issues and problems arising from Wittgenstein's philosophy of mathematics are discussed. Topics have been so chosen as to assist mediation between the perspective of philosophers and that of mathematicians on their developing discipline. There is consideration of rules within arithmetic and geometry and Wittgenstein's distinctive approach to number systems whether elementary or transfinite. Examples are presented to illuminate the relation between the meaning of an arithmetical generalisation or theorem and its proof. An attempt is made to meet directly some of Wittgenstein's critical comments on the mathematical treatment of infinity and irrational numbers.
\end{abstract}

\section{Keywords}

Wittgenstein, Philosophy of Mathematics, Rules, Number Systems, Theorem and Proof

\section{Introduction}

The philosophy of mathematics was a major concern of Wittgenstein throughout his philosophical life and he expressed the opinion at one point that his greatest contribution to philosophy might lie there (Bangu, 2018). The topic is succinctly situated within the Tractatus and was to have formed a second part of the Philosophical Investigations as originally envisaged; it was a subject taken up in his Cambridge lectures of the 1930s and his many informal writings for extended periods. Yet, it is hard to draw overall conclusions about his contribution's precise value and significance even with the help of a substantial secondary literature and also hard to disagree with Potter (2011: p. 122) that Wittgens- 
tein's impact on later philosophy of mathematics is much less than his impact on the philosophy of mind or language. The intention in this article is to draw out some strategic aspects and issues from his work in a way which enables its significance to be more readily evaluated.

It is fair to say that Wittgenstein's comments sometimes irritate mathematicians (Math-Reddit, 2013). An attempt is made here to assist a reader with only school mathematics more readily to appreciate the relation of Wittgenstein's remarks to the perspective of mathematicians themselves. What is addressed here is the account of mathematics in the Tractatus and also remarks and comments on the subject in what is generally referred to as his "middle-period" writings. Of course, awareness is needed that much of the latter derive from his notes or lectures which had not been prepared for (final) publication; nevertheless, that which is available by way of English-language publication both invites and repays our full critical attention. Among the topics taken up are rules in arithmetic and geometry, the relation between an arithmetical generalisation and its proof, and the development of number with particular attention to infinity, irrational numbers, and transfinite numbers.

Arguably, Wittgenstein does not adhere to any one of the main systematic positions within the philosophy of mathematics, logicism, intuitionism, and formalism, although his remarks are very much informed by his understanding of them. In some respects his contribution amounts to a challenge to those doctrines as well as to viewpoints that mathematicians commonly articulate themselves about the nature of, or elements of, their own subject. There is a perceptible tendency for him to say rather less about areas of mathematics which are applied either within the subject itself or within the sciences, and rather more by way of critical comment on the accounts mathematicians themselves provide of relatively isolated topics, perhaps the clearest examples being irrational and transfinite numbers.

By way of orientation, one may distinguish early, middle- and late-period writings, but with evident continuities. In the Tractatus and for most of his career Wittgenstein maintains that mathematical assertions are pseudo-propositions and that what is understood as mathematical truth is non-referential and syntactical in its nature. In what may roughly be classified as a "middle period" (of Philosophical Remarks and Philosophical Grammar) there is development towards what some have labelled "finitistic constructivism" which is subject to subsequent modification (in the Remarks on the Foundations of Mathematics). As is often commented, his writing and philosophical style which is aphoristic in the Tractatus evolves to become more argumentative in the middle period and more interlocutory in later writings. While in his middle period Wittgenstein tends to use "the calculus conception" (which includes ways of determining a quantity or required expression), later on, it is "the language-game conception" (Gerrard, 1991, 1996). In his later writings, greater sense is given to the idea that mathematical propositions are dependent upon experience. 


\section{Mathematics in the Tractatus}

Although the Tractatus is a very demanding work its stance links directly to the perspective of many educated people who understand there to be a difference between empirical generalisations requiring supporting evidence and mathematical statements requiring proof; and who also sense that the relation between mathematics and logic is particularly intimate. Wittgenstein (1922) proceeds through a contrast between contingent propositions and mathematical equations: "If the elementary proposition is true, the atomic fact exists; if it is false the atomic fact does not exist" (1922: 4.25). "The propositions of mathematics are equations, and therefore pseudo-propositions" (6.2). "And... that the propositions of mathematics can be proved means nothing else than that their correctness can be seen without our having to compare what they express with the facts as regards correctness" (6.2321). Wittgenstein understands cardinal numbers to be generated by successive applications of the operation of adding one after commencing with zero $(5.2523 ; 6.03)$. Indeed the conception of natural numbers as exponents of an operation is the hallmark of his view of arithmetic. In his account, Wittgenstein also points up the importance of applications: "In life, it is never a mathematical proposition which we need, but we use mathematical propositions only in order to infer from propositions which do not belong to mathematics to others which equally do not belong to mathematics" (6.211).

Before proceeding further it is pertinent to note the thesis of logicism which has two main components: that the concepts of mathematics can be defined in terms of logical concepts; that the theorems of mathematics may be derived from logical axioms through purely logical deduction (Savitt, 1986: p. 26). Wittgenstein seems to approach this position when strikingly he asserts: "Mathematics is a method of logic" (6.234). "The logic of the world which the propositions of logic show in tautologies, mathematics shows in equations" (6.22). Not surprisingly, therefore, some have interpreted his view as a variant of logicism but in the last analysis, this is perhaps not justified, for instance, because he does not define numbers "logically" in either Frege's or Russell's way (Rodych, 2018). He seems not to regard mathematics as reducible to logic in the manner of Whitehead and Russell in their work Principia Mathematica (Black, 1964: p. 340) and he rejects Frege's view that numbers are objects; he also firmly maintains the position that arithmetical equations are not tautologies. Furthermore, as reported by Waismann $(1979,1986)$, Wittgenstein around this time affirmed that logical operations are performed with propositions, arithmetical ones with numbers.

Potter (2011: pp. 123-124) draws attention to a specific point on which Wittgenstein departs from Russell's version of logicism. "The theory of classes", Wittgenstein writes, "is altogether superfluous in mathematics. This is connected with the fact that the generality which we need in mathematics is not the accidental kind" (6.031). He is alluding here to Russell's idea that classes may be reduced to propositional functions thus enabling one to talk about an "accidental" class, in the sense that it has as its members just those objects which happen 
to satisfy the function. This does not, however, provide a route to consideration of "essential" classes, where qualification for membership does not depend upon the properties members happen to have. However, as Potter (2011: p. 124) goes on to note, despite the evident weight given to the point, Wittgenstein fails to justify in the Tractatus his assertion that the generality needed in mathematics is not the accidental one.

Regarding significant omission in the treatment of mathematics in the Tractatus, particularly telling is the point made by Frank Ramsey (1986: p. 43) in his review of that work to the effect that the author fails to situate and deal with the role of inequalities in mathematics, as opposed to equalities. Ramsey has in mind such relations between numerical quantities or algebraic expressions as "is less than or equal to" and "is greater than" and not simply "is not equal to". One can hint at the difficulties here in the following way. Confronted with an arithmetical or algebraic equation it is very easy in language or thought to slip between the use of the words "equals" and "is", as though they were equivalents. Thus one might say "two times two equals four" or "two times two is four". The latter resembles in form such an empirically and contingently related proposition as "this patch of colour is blue". This may lead on-it may be suggested-to the philosopher of mathematics who is focusing on equations to think that the job to be performed is at least primarily to analyse the difference in the meaning and syntax (or "grammar" in Wittgenstein's sense) of types of affirmative propositions (or pseudo-propositions) across mathematics, logic and the contingent empirical sphere-at least some of which would be identities. However, it remains to be seen whether and in what way that type of analysis would bear adequately upon inequalities and the role they play in mathematics; Ramsey's probably justified presumption is that it would not.

A further significant point rather flows from Ramsey's way of putting it when he says that the pseudo-propositions of mathematics are, according to Wittgenstein, "equations obtained by writing '=' between two expressions which can be substituted for one another" (Ramsey, 1986: p. 43). This is suggestive of the idea that mathematics is concerned at least in part with equality construed as algebraic equivalence. A problem here is that mathematicians would themselves routinely distinguish between algebraic equivalence (indicated by three horizontal lines) and equality (indicated by two); the contribution of each symbol to the corpus of mathematics is different and stands in need of explication.

In the interests of completeness, it is worth adding that mathematical propositions are not exhausted by equations and inequalities. What is clear from examination of mathematical works is that the subject consists substantially of sentences and figures but there is a variety of each. Consider, for instance, the following three sentences, no one of which is an equation. If a prime $p$ divides a product $a b$, then $p$ must divide $a$ or $b$. The diagonal of a square is incommensurable with its side. If $C$ is a fixed point with coordinates $x=a, y=b$, then the locus of all points $P$ having a given distance $r$ from $C$ is a circle with $C$ as centre 
and radius $r$. It is clear that the subject is characterised by provision of proof, but it is inaccurate to say that its results and theorems simply consist of a series of equations and inequalities. Importantly, too, where an equation appears it is often embedded in a sentence containing additional words and phrases (e.g. "if... then"; "must", as above) which are essential to the argument. Certainly, it could be the case that, were all the elements in a particular theorem that do not take the form of equations to be struck out, the residue might be meaningless. Furthermore, it may well be that a consideration of the structure of mathematical reasoning as a whole-with all its various elements-would lead one back in the direction of a logicist account.

\section{The Meaning of a Statement and Its Proof}

Potter (2011: p. 127) may well be right that Wittgenstein's post-Tractatus deliberations took as a starting point the verification principle that the meaning of a proposition consists in its means of verification. Basic to mathematics is the fundamental activity of provision of proof, which may lead to the parallelsounding assertion that the meaning of a mathematical proposition is its proof. Anyway, whatever its source, we find Wittgenstein by the early 1930s making the assertion that the meaning of an arithmetical generalization consists in its inductive proof.

In this connection, suggestive is his observation that, "Generality in arithmetic is indicated by an induction. An induction is the expression for arithmetical generality" (Wittgenstein, 1975: \$149, p. 172). Even more decisive is this statement of the sense of an arithmetical generalization: "We are not saying that when $f(1)$ holds and when $f(c+1)$ follows from $f(c)$, the proposition $f(x)$ is therefore true of all cardinal numbers; but: "the proposition $f(x)$ holds for all cardinal numbers" means 'it holds for $x=1$, and $f(c+1)$ follows from $f(c)$ "' (Wittgenstein, 1974a: II, VI, §32). However, the doctrine that the meaning of an arithmetical generalization is given by its proof, as Wittgenstein recognized, gives rise to difficulties. For instance, does this mean that none of us understands the unproved statement, "Every even number is the sum of two primes" (known as Goldbach's Conjecture) prior to a proof being provided? Hence in his writing, Wittgenstein attempts to make room for the notion of a meaningful but not yet proven mathematical sentence (or conjecture) on a verificationist basis.

He makes the closely-related assertion that where a proof is laid out as a sequence of expressions, the concluding statement gets its sense from the proof and it lacks that sense apart from the proof. Thus were Goldbach's Conjecture to be proved, what we would understand at the end of the proof would differ from our present understanding. Ambrose (1986b: p. 100) puts his position thus: "an expression in the interrogative does not have the sense it has once the answer is provided; proof 'provides it with content"'.

Elsewhere Wittgenstein (1975: \$149, p. 172) slightly modifies his position by seemingly making the crucial distinction to be whether or not there is a decision 
procedure for the problem. He affirms: "Only where there's a method of solution is there a problem (of course that doesn't mean 'only when the solution has been found is there a problem')". Nevertheless one may reasonably ask how a method of solution is to be identified which has not so far generated a solution. In the absence of a solution what is the criterion for judging it to be such a method? Significant points may be drawn out from examples. It must be stressed that here one is concerned with what have been termed "open problems", i.e. outstanding problems within the mathematical community, to be sharply distinguished from problems we may encounter as individuals, e.g. a difficult long division (Säätelä, 2011: pp. 164-165).

\subsection{Example: Fermat's Last Theorem}

Wittgenstein himself refers to what is known as Fermat's Last Theorem. In interrogative form, it is asked whether, in general, for a given positive integral exponent $n>2$, the equation

$$
a^{n}+b^{n}=c^{n}
$$

can be solved with positive integers $a, b, c$. As is well known, Fermat stated that the equation is not so solvable in integers, but the elegant proof he had found was too long for the margin in which he was writing; he is believed, however, to have been mistaken. In respect of the next few centuries, one can perhaps risk the assertion that "no method of solution was identified". Only relatively recently and since Wittgenstein wrote has an actual proof been provided, by Andrew Wiles, which makes use of mathematical induction, but is very long and arduous and probably only fully understood by a handful of people. The following observation rather points against Wittgenstein's position: the proof of the theorem involves establishing the relation between elliptic curves and modular forms (Singh, 1997: pp. 183-211) but the statement of the theorem does not incorporate the associated mathematical concepts.

This controversy need not, but sometimes does, take a more personalised-sounding form as when one asks: Is what we understand as the theorem proved the same as what we understand as the theorem conjectured? A response in those terms might make a contrast between the understandings of a handful of top mathematicians with those of other members of the trained mathematical community who have not, or cannot, follow the proof. However, in sum, it is implausible that there is variation within the mathematical community as to the meaning of the generalization. This selected example is evidently one where the "gulf" between the statement of the theorem and its proof is vast.

To help make progress Ambrose (1986b: p. 102) draws attention to the possible ambiguity between proposition or statement as "sentence" or "sense of a sentence" and it may be that attention so far has been rather directed at the former. In this connection, it is worth dwelling on the position prior to the provision by Wiles of a proof. Fermat and others had conjectured that the theorem expressed a truth but no-one knew that it could not be called into question; 
conceivably a mathematician might have come forward with a counter example. The sentence might have expressed a necessary truth or, alternatively, a falsehood. Wittgenstein would probably wish to assert that a person who did not know which of these a sentence expresses did not understand by the sentence what he understands when he knows it to be true.

\subsection{Example: The Fundamental Theorem of Algebra}

Let us bear Ambrose's distinction in mind, while taking another example, seemingly relatively favourable to Wittgenstein's position. It concerns something pretty central in mathematics, what is called the Fundamental Theorem of Algebra, but it suffices here to direct attention to an example derived from it (see also Ambrose, 1986b: p. 108). The theorem is illustrated by the assertion that the polynomial

$$
f(x)=x^{4}-1
$$

may be factored into the form

$$
f(x)=(x-1)(x-i)(x+i)(x+1) .
$$

In a related way, one would say that $1, i,-i,-1$ are roots of the equation $f(x)=0$. In this connection, two of the roots are (purely imaginary) complex numbers with $I^{2}$ being -1 .

The fundamental assertion being made is that a polynomial of fourth degree such as the above necessarily has four roots (which may-as in this case-be all distinct but could be equal and would, therefore, be "counted twice or more"). However, for present purposes, a significant point is that in earlier centuries (such as the sixteenth)-prior to the introduction of complex numbersmathematicians would have firmly asserted that the above equation necessarily has two roots $(1$ and -1$)$. We can see clearly now that the apparent contradiction arises from the fact that there took place "an extension of the field of real numbers". Therefore, in a way which is supportive of Wittgenstein, one could say that in order to understand either one of "the equation necessarily has four roots" or "the equation necessarily has two roots" there is a need to understand more than either set of words as a sentence. At a minimum, one would be inclined to say that there must be an understanding of the context of the field of complex numbers or the field of real numbers, either of which would involve grasp of the mathematical concept of a field. So also must it be required that there be an understanding of what gives rise to the use of the word "necessarily". In this connection, Ambrose (1986b: p. 108) states that the necessity "did not derive from already accepted rules for the use of words, but from an extension of the use of 'root'". Hence there is a discernible tendency for seemingly straightforward "understanding of a sentence" to "unravel" and the "direction of travel" is towards understanding important elements involved in the proof.

\subsection{Example: A Specified Sequence in a Decimal Expansion}

Let us take one further example, of a type considered by Wittgenstein. Suppose it 
is asked: Does the sequence 66 occur in the decimal expansion of 1/7? It must be stressed that the decimal expansion fails to terminate, hence it may initially appear that one is being invited to look for a needle in an infinite haystack, which would be an absurd way to proceed.

Suppose, to make progress, one commences determining the decimal expansion by long division. After say ten or more decimal places one may perhaps notice that a sequence of digits is repeating; in addition, that sequence does not include the digit six. Hence one begins to lean to the view that one is presented with an endlessly recurring set of digits which does not include a six. However, the crucial next step is to determine for sure i.e. prove that the particular set of digits is indeed endlessly recurring. This would entail either developing an ad hoc proof oneself or citing a more general result of which this is a particular instance. The important general mathematical result is that any rational number, that is any number which can be expressed in the form $p / q$, where both $p$ and $q$ are integers, can be expressed as a finite or as an infinite recurring decimal. This decimal need not be a pure recurring decimal, but after a finite number of digits the decimal must recur. Familiarity with this result transforms the situation. Either way, there is evidently some substance in Wittgenstein's idea that the answer to an expression in the interrogative "provides it with content". This falls short, however, of justification for the assertion that one can understand the meaning of an expression or result only through understanding of its proof.

\section{The Development of Number and its "Grammar"}

In addition to his concern with the relation of meaning and proof, in his middle period Wittgenstein directed a lot of attention to the number concept and, in particular, to issues to do with the infinite. Adopting a more argumentative style, he is led to challenge aspects of the latter notion as used by mathematicians; especially what he terms "infinite mathematical extensions". The intention here is to track both the predominant approach of mathematicians and his critical view, so as to assist an evaluation. In this connection it is important to direct attention both towards the nature of mathematical propositions (or pseudo-propositions) and also to the development of the number concept. A good example of change in the number concept has in fact already been alluded to, for, in the last section, reference is made to the emergence of complex numbers. The issues are perhaps best approached via the most basic and even elementary-seeming concerns.

In his Remarks on the Foundations of Mathematics Wittgenstein situates mathematical verification and relates it to the determination of meaning; this links to his doubts about infinite mathematical extensions. True propositions within mathematics have that status because they are provable in a calculus. They are deductions from axioms by formal rules and are true by virtue of valid application of the rules of inference; they owe nothing to the world outside mathematics. The key point is that a mathematical calculus is a calculus for the transformation of signs. However, as Goodstein puts it, "mathematics is not a mere 
game, because the natural numbers have a use outside mathematics, and this use of numbers in ordinary language is what gives mathematics its meaning". (Goodstein, 1986: p. 282, italics in original)

In an oft-quoted passage, Wittgenstein says, "it is essential to mathematics that its signs are also employed in mufti. It is the use outside mathematics, and so the meaning of the signs, that makes the sign-game into mathematics" (Wittgenstein, 1978: V, p. 257). The equation $2+3=5$ is a derivable consequence of the definition of addition in the sign calculus of mathematics; but if the world changed so that it ceased to be the case that whenever we placed three eggs in a box which already contained two eggs then only four eggs remained instead of five, the equation would retain its mathematical validity, but lose its application in the world. The failure to find a use for the equation outside of mathematics would deprive it of meaning. Mathematics is applied but not verified in the world (Goodstein, 1986: pp. 282-283).

\section{Some Developmental Stages for Number}

Moving next to the overall developmental picture for number-particularly as this is understood by mathematicians-one may perhaps say that the concept has "evolved" where this is meant in the original sense of "unfolding". Originating with the natural numbers, systematic development has occurred subject to the principle of generalisation; at the same time, the discipline of mathematics has been integrated by the coming together of algebra and geometry within analytical geometry. There is a perceptible passage towards "completeness" the source of which becomes clear as particular stages are briefly adumbrated.

In the arithmetic of the natural numbers one can always carry out the two fundamental operations of addition and multiplication, but the "inverse operations" of subtraction and division are not always possible. The steps towards removing the restriction were taken when the symbol 0 and the symbols for negative numbers were introduced. This ensures that subtraction may be performed without restriction in the domain of positive and negative integers. It is necessary in this connection to define operations in such a way that the original rules of arithmetical operations are preserved. Thus the initially strange-seeming rule $(-1)(-1)=+1$ is a consequence of the desire to preserve the distributive law of multiplication $a(b+c)=a b+a c$.

What has just been said may be contrasted with elements of Wittgenstein's distinctive perspective. He would have been aware that when negative numbers first made an appearance-to handle debits as well as credits-as numbers they were greeted with the same abhorrence as were "imaginary" numbers later on; that abhorrence arises from violation of the existing syntax or "grammar" associated with natural numbers. In the natural number system it makes no sense to subtract a larger from a smaller number. The integers constitute a new number system and it is misleading to consider that as an expansion of an old number system. Associated with a new numerical series is a new grammatical system 
which is such that analogues of previously unintelligible questions acquire meaning and become soluble. What happens is that a new system "extends the family of systems gathered under the concept of 'number" (Shanker, 1986: p. 9).

One may briefly track the further development of number: the introduction of fractions removes the analogous arithmetical obstacle to division; rational numbers are provided with a geometrical interpretation enabling the magnitudes of line segments to be compared; analytical geometry enables every geometrical object and operation to be referred to the realm of numbers. Each of these systems has its associated grammar. A further development concerns the definition of the real number system and irrational numbers. Since this last is a focus of many of Wittgenstein's comments slightly more detail is provided here.

Numbers may be expressed to any whole number base greater than one but the reader will be familiar with the fact that we customarily express numbers to base ten and also use place value. Furthermore one can cover the number axis with those numbers which originate by subdivision of each unit interval into 10, then 100,1000 , etc. equal segments. The points so obtained correspond to "decimal fractions" and give rise to the familiar representation of a number in the decimal system by the abbreviated symbol $z \cdot a_{1} a_{2} a_{3} \ldots a_{n}$. Informally, one then proceeds to define the continuum of real numbers or real number system ("real" in contrast to "complex" numbers referred to earlier) as the totality of infinite decimals. As indicated in an earlier section all rational numbers are either finite or periodic decimals. The irrational numbers are the non-periodic decimals. (In a more formal development of the topic an irrational point on the number axis may be defined as a symbol for a certain sequence of nested rational intervals with lengths tending to zero. There are other equivalent approaches to that definition including the use of "Dedekind cuts" mentioned below.)

\section{Infinity and Irrational Numbers}

A key difference between the early and middle Wittgenstein is that, at the later stage, he rejects quantification over an infinite domain. Strategically important in this connection are his conception of mathematics as a human invention and the notion that mathematical calculi are exclusively made up of what he terms "intensions" and "extensions". His usage is unconventional: by "extensions" he means such items as symbols, axioms, propositions, and finite sequences, while by "intensions" he has in mind rules of differing kinds, such as rules of inference.

Wittgenstein (1975: $\$ 144$, p. 164 ) casts doubt on the idea of actual infinite sets: "The infinite number series is only the infinite possibility of finite series of numbers. It is senseless to speak of the whole infinite number series, as if it, too, were an extension". Additional comments of his bear upon the distinction between the actual and the potential infinite. He argues more directly against the existence of infinite sets as follows:

Let's imagine a man whose life goes back for an infinite time and who says 
to us: "I'm just writing down the last digit of $\pi$ and it's a 2." Every day of his life he has written down a digit, without ever having begun; he has just finished.

This seems utter nonsense, and a reduction ad absurdum of the concept of an infinite $\operatorname{totality}(1975: \$ 145$, p. 166).

Taken literally this is unsatisfactory since $\pi$ is irrational (known since the eighteenth century), its decimal expansion does not terminate, so it has no last digit. To say that the expansion "has no last digit" is a clear idea which has nothing to do with time. Therefore its clarity cannot be challenged by way of an imaginary exercise involving performance in time.

There are indeed several points in his writings where Wittgenstein appears to query the actual infinite (Wittgenstein, 1978: V, \$21) and infinite mathematical extensions, which has given rise to discussion as to whether his approach may fairly be characterised as "finitist". Indicative is his claim that, "[t]he concepts of infinite decimals in mathematical propositions are not concepts of series, but of the unlimited technique of expansion of series" (Wittgenstein, 1978: V, \$19). He also affirms that, "[t] he extensional definitions of functions, of real numbers etc. pass over-although they presuppose-everything intensional..." (Wittgenstein, 1978: V, \$35). He asserts that irrational numbers are rules for constructing finite expansions, not infinite mathematical extensions. Alluding to the method of defining irrational numbers by the use of "Dedekind cuts", he queries whether we are "led to $\sqrt{ } 2$ by way of the concept of a cut" and characterises the cut as "an extensional image" (1978: V, \$34). Of critical importance is his claim that there is no rule, no systematic means of defining each and every irrational number intensionally, which leads him to question whether there is a criterion "for the irrational numbers being complete" (1975: \$181, p. 223).

\section{Irrational Numbers: Definition; Examples; A Criterion for Completeness}

What Wittgenstein appears to mean by defining an irrational number "intensionally" is illustrated by the definition of $\sqrt{2}$ as the length of the diagonal of a unit square, and $\pi$ as the length of the circumference of a circle of unit diameter. Both definitions are geometrical on their face, but one must bear in mind the geometrical interpretation of number noted earlier. On the other hand, what Wittgenstein has in mind by a "mathematical extension" would appear to be illustrated in the present context by the representation of a quarter by 0.25 ; where the two digits after the decimal point are specified and ordered. What he would appear to be objecting to in an "infinite mathematical extension" such as the representation of $\sqrt{2}$ as $1.414 \ldots$ is that only some of the digits are individually identified and ordered. Part of the response is to say that, if anyone cares to specify any arbitrary whole number (such as a hundred), a further extension may in principle be provided to reveal further digits up to the specified whole number of decimal places. The other part of the response is to say that it is not possible 
to specify a last digit because there is no last digit.

It is important to make more precise what is being said about $\sqrt{2}$ and $\pi$. The basic assertion is that each gives rise to an indefinitely extended decimal fraction. In the first case no explicit formula is known to determine the digits but one may calculate as many as one wishes. In the second case there are formulas which enable $\pi$ to be expressed as an infinite series, from which its decimal expansion may be determined to any required degree of accuracy. Thus each may be shown as an indefinitely extended decimal fraction of the form $z \cdot a_{1} a_{2} a_{3} \ldots$, where the dots indicate that the decimal fraction may be extended "indefinitely". This implies that were someone to require an extension to an arbitrary whole number of decimal places it could in principle be provided. $\sqrt{2}$ and $\pi$ are irrational numbers, but it is pertinent to add that many rational numbers give rise to an indefinitely extended decimal expansion albeit one which is periodic, the most familiar being the expression for a third as $0.333 \ldots$ Thus there is a perfectly clear sense in which the real numbers-or the "real number continuum"-may be considered as "complete", and within that context, $\sqrt{2}$ and $\pi$ belong to the irrationals - the non-periodic decimals-which may also be considered as complete.

Wittgenstein is critical of a tendency to employ metaphors deriving from geography e.g. the claim that irrational points fill the "gaps" on a line left by the rational points, just as we might claim that certain sediments fill the gaps in a rock left by igneous material. No responsible mathematician would do this, however: for one thing a "gap" would be a measurable interval, while an indefinitely large number of rational numbers is found within any interval, however small. Again, he writes: "We are surprised to find that 'between the everywhere dense rational points' there is still room for the irrationals. (What balderdash!)" (Wittgenstein, 1974a: p. 460; also $\$ \$ 40-1$ ) No-one is claiming, however, that any "room"-or interval-is either needed or available to "accommodate" irrationals: as noted above, each is being defined as the "limit" of an indefinitely large sequence of rationals (or of a sequence of nested intervals with rational endpoints). In the latter connection, there may well be employment of such formal phraseology as "the limit as $\mathrm{n}$ tends to infinity" but this is used rather as a "term of art" (c.f. legal terminology): it carries absolutely no implication that infinity is to be treated like a natural number.

In seeking to identify a criterion for the irrational numbers being complete Wittgenstein argues as follows:

Suppose we had the totality of all irrational numbers with one single exception. How would we feel the lack of this one? And if it were to be added, how would it fill the gap? Suppose that it's $\pi$. If an irrational number is given through the totality of its approximations, then up to any point taken at random there is a series coinciding with that of $\pi \ldots$ And so if I have the totality of all irrational numbers except $\pi$, and now insert $\pi$, I cannot cite a point at which $\pi$ is now really needed. At every point it has a companion 
agreeing with it from the beginning on. This shows clearly that an irrational number isn't the extension of an infinite decimal fraction, that it's a law.

Our answer to the question above must be "If $\pi$ were an extension, we would never feel the lack of it", i.e. it would be impossible for us to observe a gap. If someone were to ask us, "But have you then an infinite decimal expansion with $m$ in the $r$-th place and $n$ in the $s$-th place, etc.?" we could always oblige him (Wittgenstein, 1975: XVII, $\$ 181$, p. 223).

So Wittgenstein's argument centres upon whether we would feel the lack of a particular irrational number such as $\pi$. As indicated earlier, however, it is quite inappropriate to speak as though $\pi$ "fills a gap" because a gap is a measurable interval. Nevertheless, since an irrational number is defined as the limit of an infinite series of rational numbers, it follows directly that, as indicated by Wittgenstein, one could supply an infinite decimal expansion which coincides with that of $\pi$ up to a specified point. However, it would also be the case that presented with any such infinite decimal expansion, one could shows its divergence from that of $\pi$ at some subsequent point. This could be done because, as indicated earlier, the expansion of $\pi$ is given by a formula and hence its decimal expansion may be extended to any required degree. It may be worth adding that the digits in that expansion exhibit no pattern: in a certain sense, they have a random character. Hence, no clear sense may be given to the notion of law in respect of that decimal expansion. Yet the very fact that there are formulae giving its value to any required number of decimal places identifies and secures its unique place within the decimal expansions of irrational numbers.

Given that the real numbers are closed with respect to the rational operations, a further twist may be given to the argument. Suppose we take the infinite series which sums to the value of $\pi$ and simply delete the first term. Then the sum of the residual series would sum to an irrational number with a value different from that of $\pi$. In addition, the deleted term, as a rational number, would give rise to a further decimal expansion. Suppose these two decimal expansions are now summed then the resultant is the value of the decimal expansion of $\pi$. Hence if the latter decimal expansion were considered as absent, we would feel the lack of it because there would be a violation of the rule that the real numbers are closed under addition. Hence it is the rule concerning closure under the rational operations that underlies the claim that the irrational numbers are complete.

Particularly given Wittgenstein's sensitivity to applications, it may be wondered whether irrational numbers are of use outside mathematics. Doubts may be raised because it is impossible to determine by any physical operation whether a given length is rational or irrational. What is true, though, is that, from a physical point of view, the definition of a real number by a convergent series of rational numbers corresponds to the determination of the value of some observable quantity by a sequence of measurements of greater and greater accuracy. Therefore, as Courant and Robbins (1958: p.71) argue, "the real advantage 
which the introduction of irrational numbers brings to the mathematical description of physical phenomena is that the description is enormously simplified by the free use of the limit concept, for which the number continuum is the basis".

\section{Transfinite Numbers and the "Excluded Middle"}

Wittgenstein addresses issues concerning infinity and denumerability which are probably more readily appreciated given a (necessarily brief) presentation of context. Now considering the infinite class of the natural numbers

$$
123456 \ldots n \ldots
$$

one can set up a one-to-one ("biunique") correspondence between these numbers and the series obtained by doubling each of them:

$$
24681012 \ldots 2 n \ldots
$$

Following Cantor, one defines an infinite class as one which can-as in this case-be put into one-to-one correspondence with a part (or proper subset) of itself. In addition, if two infinite classes are such that a one-to-one correspondence can be set up between their members, then it is said that the two classes have the same transfinite number of members. Where a class has the same transfinite number as that of the natural numbers one says that it is denumerable or countable. Cantor went on to establish some further surprising results. $\mathrm{He}$ showed-by a constructive and systematic method-that the class of all positive rational numbers is countable. On the other hand, by an indirect method-and using a "diagonal process" - he showed that the class of all real numbers between 0 and 1 is not denumerable. This leads on to the assertion that the transfinite number of the latter class is greater than that of the class of natural numbers. Importantly for present purposes Cantor's indirect method is a proof by reduction ad absurdum i.e. it proceeds by assuming the contrary and then showing that this leads to a contradiction. The school of intuitionists does not accept proofs of that type and Wittgenstein is far from being impressed by it (Moore, 2019a).

Importantly, Wittgenstein (1974b: p. 124) tells us: "Philosophy may in no way interfere with the actual use of language", "it can in the end only describe it... It leaves everything as it is. It also leaves mathematics as it is". Yet in challenging the terms in which mathematical results are formulated, he sometimes seems to challenge the results themselves (Moore, 2011: p. 117). Thus Wittgenstein (1975: $\$ 141$, p. 161) queries whether the relation $m=2 n$ can be used to correlate the set of natural numbers with one of its own proper subsets, in the way just indicated. Again, he writes: “one pretends to compare the 'set' of real numbers in magnitude with that of cardinal numbers... I believe, and hope, that a future generation will laugh at this hocus pocus" (Wittgenstein, 1978: II, \$22).

Those-such as Wittgenstein-who object to the type of non-constructive reasoning employed by Cantor focus inter alia on its character as an essentially indirect proof. As above, the form of an indirect proof follows this pattern: to 
establish the truth of a statement, one makes the provisional assumption that its contrary is true. Then by a chain of reasoning one produces a contradiction to the contrary statement, thus demonstrating its absurdity. Then on the basis of the logical principle of the "excluded middle" the absurdity of the contrary statement is taken to establish the truth of the original statement.

Now Wittgenstein is conscious of ways in which mathematicians may import conceptual confusion into their discipline, for instance, as Moore (2011: p. 118) puts it, by their "unthinking commitment ...to the law of the excluded middle... another symptom of an unwarranted realism". Importantly, one must note that in mathematics there are examples where an indirect proof can be converted into a direct proof, but there are also theorems - such as Cantor's as given earlier on real numbers-for which direct constructive proofs could not possibly be given even in principle. There is little doubt that the complete banishment from mathematics of non-constructive proofs would constrict the growth of the subject.

Wittgenstein is particularly helpful when directing critical attention to Cantor's claim that the transfinite cardinals constitute, "an extension of the concept of number" (quoted Shanker, 1986: p. 5). As Shanker notes, this phrase may be interpreted in either of two ways: an extension of the number concept in itself; an extension of the domain of the natural numbers. Wittgenstein would find the first claim acceptable since he viewed number as a "family resemblance" concept. The second interpretation is much more dubious. While in conventional terms, there is sense to the notion that the domain of the natural numbers may be extended into that of positive and negative integers and on into the real numbers, the notion that transfinite numbers derive from a similar extension is untenable. In the former case the extensions are all to do with preserving and extending the use of the rational operations, but in the latter case there is no such preservation of operations. Just about the only "operations" which are specified for Cantor's transfinite numbers are "equal to" and "greater than" (and the latter hardly works in the same manner as $2>1$ ): you cannot multiply or divide with them!

As already noted, for Wittgenstein suitable application is the hall-mark of meaningful mathematical activity. This particular arrow could be targeted on transfinite mathematics which has virtually no use in non-mathematical contexts. Nevertheless, as Moore (2011: p. 119) points out, one may ask such questions as: Why need the application be external to mathematics? Why may not the development of its application lie in the future (as complex numbers found application in alternating current theory of electricity)? Even lacking application, might it not usefully systematize other parts of mathematics? One must be sure never to underrate the complexity of both the internal and external connections of branches of mathematics.

\section{Rules within Arithmetic and Geometry}

The contrast with empirical propositions is again foregrounded in Wittgens- 
tein's middle period writings. Motivated by an analogy with those propositions, people are inclined to ask, "What are mathematical propositions about?", but against this tendency Wittgenstein forwards the suggestion that arithmetical propositions (or pseudo-propositions) are not about numbers, nor are geometric propositions about geometrical figures. According to him, we would do well to direct our attention away from what they are about and towards the use which we make of them. Furthermore, in a key point which Ambrose (1986a: p. 26) draws from his work, where we allow nothing to count against a proposition, the expression for the proposition is being assigned a special role in the language, as a rule for the application of certain words.

This may be illustrated by our orientation in the familiar type of tiling situation. Suppose one multiplies 13 by 12 to find out the number of unit squares needed to exactly cover a rectangle 13 units long by 12 units wide. Were one to find upon counting that the number of tiles needed is other than 156, the result obtained by multiplication according to the rules, one could conceivably respond in two different ways. Were one to say " $13 \times 12=156$ is false", one would be treating it as a proposition testable by experience. On the other hand, were one to say that the multiplication is correct regardless of the number of unit squares counted, one would be using it as a criterion as to the correctness of the count, and hence independent of experience (Ambrose, 1986a: p. 26). The second is our established, common linguistic practice.

As Ambrose (1986a: p. 28) explains, Wittgenstein's account helpfully bears upon the familiar puzzle, that people sometimes seem to discover a fact of experience which they then go on to prove must be so. This would seem to give a matter of fact an a priori demonstration. The assertion that there are only five regular polyhedra would be a case in point; this proposition was believed centuries before it was actually proved. However, according to Wittgenstein, what is happening is that an empirical proposition arising out of experience is converted into a proposition which no experience would lead us to give up. As Ambrose (1986a: p. 30) puts it, “...arithmetical propositions, though independent of experience, are in two ways dependent upon experience: in being suggested by experience, and in having their special function rest on common linguistic habits". Furthermore, consideration of the relation between mathematical proof and experience rather dramatises Wittgenstein's conception of mathematical proofs as paradigms.

There would, however, seem to be a significant difference between arithmetic and pure geometry in relevant respects. In the latter case, but not in the former, there is a discernible tendency of linguistic practice to assert that something is true of "ideal" elements-such as geometrical lines and circles; it is implied that it may not precisely be the case, for instance, for lines or circles drawn on paper. Thus in pure geometry, the proposition treated as independent of experience is that contained within the abstracted axiomatised system. Pure geometry proceeds against the background of continued understanding that there is a gulf 
between the world of experience and the abstracted model, even given the illumination that each provides of the other. It is that understanding which eventually opens the way to the substantive question as to whether Euclidean or non-Euclidean geometry is to be preferred as a description of the geometry of the physical world.

Wittgenstein was fascinated by proofs of impossibility, for instance, those directed at problems in ancient Greek geometry concerning the straightedge and compass trisection of an angle, squaring of the circle, and construction of a regular heptagon. In the last two centuries, the impossibility of each of these constructions has been demonstrated by algebraic methods. However, within the framework of geometrical straightedge and compass construction itself, no such demonstration of impossibility could be provided. It is only within a radically different algebraic setting that the problem can be properly posed and solved. Wittgenstein is firm on the point that it makes no sense to talk of the nonexistence of a construction, in the context of constructions. Modern algebra and algebraic geometry provide a new grammatical system and associated rules within which questions unintelligible within the system of ancient Greek geometry acquire meaning and become soluble.

\section{The Rule-Following Argument}

Particularly well-known-and notoriously difficult satisfactorily to rebut-is Wittgenstein's "rule-following argument", to the effect that each time we apply a rule there is in some sense an indeterminacy as to what "applying the rule" amounts to on this occasion. An example taken for consideration is where an individual appears to be "successively adding two" but then in their determinations, they suddenly diverge from what an observer expects. It is relevant to say that given a mathematical series more than one rule may generate its early members but then give different values at a later point. As presented, Wittgenstein's argument is entirely general, so any sense of context tends to be lost. Is it helpful-one may wonder-to consider a young child learning to count, or the limited arithmetic of a tribal society, or present day higher mathematics?

In fact, further to the discussion of the solutions of quartic equations above, one can even construct an imaginary example from higher mathematics which may be illustrative. Suppose, as a twenty-first century mathematician, one is overlooking the work of a sixteenth century mathematician who is seemingly recording the number of roots of a series of quartic equations (making due allowance for repeated roots). He records an extended series of fours and we may be led to expect this to continue but then-all of a sudden-he records a two.

Where an individual says they are following a rule which they may or may not identify explicitly, or where they appear to be following a rule, and they then act in an unexpected way, one may seek "to go behind it"-by asking them to explain their choice or decision, or by investigating it indirectly. In the example just given it might thereby be quickly revealed that only "real" roots are being 
counted. Again, supposing the context is of a young child learning to count, one might have recourse to the use of counting beads or Cuisenaire Rods. Importantly, there is nothing in Wittgenstein's example that prevents an individual subsequently being led to (or independently arriving at) an alternative choice or the genuinely-held judgment that they had previously "made a mistake"; but arguably, there is also nothing that compels them to do so either. Again, while taking Wittgenstein's $(1975, \$ 164$, p. 198) point regarding "the unbridgeable gulf between rule and application, or law and special case" little or nothing follows from it regarding the extent to which, or contexts within which, unresolvable issues of that type may be expected to be encountered in practice.

In connection with arithmetic or any other part of mathematics what is crucial is not that we are capable of coming to differing judgements or assessments (as when we repeatedly check bank statements) but the recognition that there is an objectively given criterion as to the correctness of the count, the sum or the relevant calculus. Significantly, the use of such objective criteria enters into the design of the aids we employ such as calculators and computers, which therefore serve to discipline our behaviour. The nature of Wittgenstein's "unbridgeable gulf" could be such that the majority of a population fails to recognise a standard of correctness and/or their own regular departure from it. That this is not the case and that we recognise the possibility of our own mistakes and struggle to correct them lies at the heart of the institution of mathematics within society. Mathematical rules can only be said to function where they are sustained within common linguistic practice: happily the position in society today.

\section{Concluding Remarks}

In a strikingly impressive way the Tractatus lays bare the essentials in terms of abstraction and generality. Within the account, Wittgenstein characterises and situates mathematical propositions (or pseudo-propositions) while departing from Russell's and Frege's versions of logicism. However, given that important work's character and aspirations, one is entitled to ask whether in its own gnomic terms it "does justice" to mathematics. Doubts in this respect centre on narrowness in its characterisation of the subject matter given a focus on the mathematical equation. The reader is rather left wondering how Wittgenstein would situate not just the inequalities cited by Ramsey, but a variety of other propositions. An extension of Wittgenstein's analysis to incorporate the wider structure of mathematical reasoning might well lead one back towards a logicist account.

With the help of examples, the relation between the meaning of an arithmetical generalization and its inductive proof has been explored, but one is unable to conclude that the former consists of the latter. To be fair to Wittgenstein it may be that his doctrine is to be interpreted narrowly-as being solely concerned with mathematical induction - and does not even extend to include the fundamental theorem of algebra considered here; but there is considerable interest in 
examining the relation more generally within mathematics. In marked contrast in relevant respects to Fermat's Last Theorem is the Fundamental Theorem of (Integral) Calculus. In that case, extended study is needed to gain understanding of the context, preliminaries and formulation of the theorem, but the increment of understanding then needed to grasp the (perhaps two sentence) proof is relatively slight (Apostol, 1957: p. 215): meaning of general proposition and of its proof are evidently close.

The attempt is made here to meet directly some of Wittgenstein's objections to the mathematical treatment of infinity and irrational numbers (Moore, 2019a). Infinite processes play a central role in the subject, particularly in differential and integral calculus, its "crowning glory". It is agreed among mathematicians that the analytical work of the nineteenth century-by Weierstrass and others - provides a perfectly sound basis, yet it is of the essence that only a finite number of steps are taken in that analytical reasoning. As Wittgenstein (1974a: p. 483) reminds us: "Let us not forget: mathematicians' discussions of the infinite are clearly finite discussions. By which I mean, they come to an end" (see Moore, 2019a, 2019b). To challenge their approach would simply require a challenge to one or more of a finite number of steps; but no such challenge is evidently forthcoming. There is thus a sense in which the infinite is satisfactorily addressed by the finite; yet, emphatically, this need not involve adherence to a doctrine of "finitism".

Wittgenstein's comments on the place of transfinite numbers within mathematics are informed by considerations similar to those he judges to apply to more elementary-seeming extensions of the number concept, for instance, that from the natural numbers to the integers. His view is that we are presented with a further extension of the family of systems subsumed under the concept of number. There is an important difference to note, however. The extensions from natural numbers through to the real number continuum are all to do with extending the use of the rational operations while preserving the rules. (The extension to complex numbers similarly preserves operations and rules while systematising and completing the solutions of algebraic equations.) Any supposed "extension" to transfinite numbers is, however, of an entirely different order, partly because there is no preservation of rational operations and the associated rules. Hence a challenge to the claim that the introduction of transfinite numbers extends the domain of the natural or the real number systems is wellfounded.

Wittgenstein surely rightly values absolute clarity and also, in a not unfamiliar way, sees merit in the potentially creative perspective of the young child:

Philosophical clarity will have the same effect on the growth of mathematics as sunlight has on the growth of potato shoots (In a dark cellar they grow yards long).

A mathematician is bound to be horrified by my mathematical comments, since he has always been trained to avoid indulging in thoughts and doubts 
of the kind I develop...I trot out all the problems that a child learning arithmetic, etc. finds difficult, the problems that education represses without solving. I say to those repressed doubts: you are correct, go on asking, demand clarification! (Wittgenstein, 1974a: pp. 381-382).

While sympathising with the general sentiment, one is bound to say, further to the above discussion of the "unfolding" concept of number, that the child may not experience accurate answers as particularly satisfying. For instance, everyone finds initially strange the rule $(-1)(-1)=+1$, but to be told that "this is true by definition" or "is a rule needed to preserve the distributive law of multiplication" is unlikely to bring comfort to the budding mind. The combination of simplicities and complexities within the evident richness of mathematics is pretty unfathomable to us all.

\section{Conflicts of Interest}

The author declares no conflicts of interest regarding the publication of this paper.

\section{References}

Ambrose, A. (1986a). Wittgenstein on Some Questions in Foundations of Mathematics. In J. V. Canfield (Ed.), The Philosophy of Wittgenstein: Volume 11, Philosophy of Mathematics (Chapter 2, pp. 21-38). London: Garland.

Ambrose, A. (1986b). Proof and the Theorem Proved. In J. V. Canfield (Ed.), The Philosophy of Wittgenstein: Volume 11, Philosophy of Mathematics (Chapter 6, pp. 99-109). London: Garland.

Apostol, T. M. (1957). Mathematical Analysis: A Modern Approach to Advanced Calculus. Reading, MA: Addison-Wesley.

Bangu, S. (2018). Ludwig Wittgenstein: Later Philosophy of Mathematics. In Internet Encyclopedia of Philosophy. http://www.iep.utm.edu/wittmath/

Black, M. (1964). A Companion to Wittgenstein's Tractatus. Cambridge: Cambridge University Press.

Courant, R., \& Robbins, H. (1958). What Is Mathematics? London: Oxford University Press.

Gerrard, S. (1991). Wittgenstein's Philosophies of Mathematics. Synthese, 87, 125-142. https://doi.org/10.1007/BF00485331

Gerrard, S. (1996). A Philosophy of Mathematics between Two Camps. In H. Sluga, \& D. G. Stern (Eds.), The Cambridge Companion to Wittgenstein (pp. 171-97). Cambridge: Cambridge University Press. https://doi.org/10.1017/CCOL0521460255.006

Goodstein, R. L. (1986). Wittgenstein's Philosophy of Mathematics. In J. V. Canfield (Ed.), The Philosophy of Wittgenstein: Volume 11, Philosophy of Mathematics (Chapter 12, pp. 269-284). London: Garland.

Math-Reddit (2013). What Do Mathematicians Think about Wittgenstein? https://www.reddit.com/r/math/comments/1dcrx3/what_do_mathematicians_think_ab out_wittgenstein/

Moore, A. W. (2011). Wittgenstein and Infinity. In O. Kuusela, \& M. McGinn (Eds.), The Oxford Handbook of Wittgenstein (Chapter 5). Oxford: Oxford University Press. https://doi.org/10.1093/oxfordhb/9780199287505.003.0006 
Moore, A. W. (2019a). The Infinite (3rd ed.). London: Routledge. https://doi.org/10.4324/9781315145921

Moore, A. W. (2019b). Language, World and Limits: Essays in the Philosophy of Language and Metaphysics. Oxford: Oxford University Press.

Potter, M. (2011). Wittgenstein on Mathematics. In O. Kuusela, \& M. McGinn (Eds.), The Oxford Handbook of Wittgenstein (Chapter 6). Oxford: Oxford University Press. https://doi.org/10.1093/oxfordhb/9780199287505.003.0007

Ramsey, F. (1986). Critical Notice of the Tractatus. In S. Shanker (Ed.), Ludwig Wittgenstein, Critical Assessments (pp. 34-46). London: Croom Helm.

Rodych, V. (2018) Wittgenstein's Philosophy of Mathematics. In E. N. Zalta (Ed.), The Stanford Encyclopedia of Philosophy (2018 ed.). https://plato.stanford.edu/archives/spr2018/entries/wittgenstein-mathematics/

Säätelä, S. (2011). From Logical Method to "Messing about": Wittgenstein on "Open Problems" in Mathematics. In O. Kuusela, \& M. McGinn (Eds.), The Oxford Handbook of Wittgenstein (Chapter 8). Oxford: Oxford University Press. https://doi.org/10.1093/oxfordhb/9780199287505.003.0009

Savitt, S. (1986). Wittgenstein's Early Philosophy of Mathematics. In S. Shanker (Ed.), Ludwig Wittgenstein, Critical Assessments (Vol. 3, pp. 26-35). London: Croom Helm.

Shanker, S. (1986). Introduction: The Portals of Discovery. In S. Shanker (Ed.), Ludwig Wittgenstein, Critical Assessments (Vol. 3, pp. 1-25). London: Croom Helm.

Singh, S. (1997). Fermat's Last Theorem. London: Fourth Estate.

Waismann, F. (1979). Wittgenstein and the Vienna Circle. Oxford: Blackwell.

Waismann, F. (1986). The Nature of Mathematics: Wittgenstein's Standpoint. In S. Shanker (Ed.), Ludwig Wittgenstein, Critical Assessments (Vol. 3, pp. 60-67). London: Croom Helm.

Wittgenstein, L. (1922). Tractatus Logico-Philosophicus. London: Routledge \& Kegan Paul.

Wittgenstein, L. (1974a). Philosophical Grammar. Oxford: Blackwell.

Wittgenstein, L. (1974b). Philosophical Investigations. Oxford: Blackwell.

Wittgenstein, L. (1975). Philosophical Remarks. Oxford: Blackwell.

Wittgenstein, L. (1978). Lectures on the Foundations of Mathematics, Cambridge, 1939. Ithaca, NY: Cornell University Press. 